\title{
Ondas de Alfvén no meio interplanetário
}

(Alfvén Waves in interplanetary space)

\author{
E. Costa Jr. ${ }^{1,3}$, F.R. Cardoso ${ }^{3}$, F.J.R. Simões Jr. ${ }^{2,3}$ e M.V. Alves ${ }^{3}$ \\ ${ }^{1}$ Instituto Federal de Minas Gerais, Ouro Preto, MG, Brasil \\ ${ }^{2}$ Departamento de Física, Fundação Universidade Federal de Pelotas, Pelotas, RS, Brasil \\ ${ }^{3}$ Laboratório Associado de Plasma, Instituto Nacional de Pesquisas Espaciais, S.J. dos Campos, SP, Brasil
}

Recebido em 19/4/2010; Aceito em 24/2/2011; Publicado em 3/6/2011

\begin{abstract}
Ondas de Alfvén são ondas transversais que se propagam ao longo das linhas de campo magnético e podem ser geradas em qualquer fluido eletricamente condutor permeado por um campo magnético. Foram deduzidas em 1942 por Hannes Alfvén através das equações do eletromagnetismo e da hidrodinâmica. A confirmação experimental de sua existência ocorreu sete anos depois, através do estudo de ondas em mercúrio líquido. Já se sabe que essas ondas constituem um mecanismo importante de transporte de energia e momentum em vários sistemas hidromagnéticos, tanto astrofísicos quanto geofísicos. Foram observadas em plasmas interplanetários, na fotosfera solar e na magnetosfera terrestre, onde podem causar as atividades magnéticas chamadas HILDCAAs. Pela sua alta ocorrência e pelo seu papel na ligação e troca de informações entre regiões distintas do espaço, constituem um campo de investigação de interesse crescente, tanto através do seu estudo no meio interplanetário e em laboratórios, quanto por simulações computacionais.
\end{abstract}

Palavras-chave: física espacial, ondas de Alfvén, teoria MHD.

Alfvén waves are transversal waves that propagate along magnetic field lines. They can be generated in any fluid able to conduct electricity and in the presence of a magnetic field. The waves were matematically discovered in 1942 by Hannes Alfvén using electromagnetic and hidrodinamic equations. Experimental confirmation of their existence took place seven years later by investigations about waves in liquid mercury. It is known that these waves are an important mechanism of energy and momentum transport in many hidromagnetic system, both astrophysical and geophysical. The waves have been observed in interplanetary plasmas, at the solar photosphere and terrestrial magnetosphere, where they can lead to magnetic activities called HILDCAAs. Due to their high level of ocurrence and important role in the connection and exchange of information between different regions of space, the waves constitute an important field of study, through their investigation in the interplanetary space and laboratories and also by computational simulations.

Keywords: space physics, Alfvén waves, MHD theory.

\section{Introdução}

Graças ao progresso da física e das ciências espaciais ocorrido no século passado, foi descoberto que a maior parte da matéria conhecida no universo é formada por plasmas [1]. Podendo ser entendido como o quarto estado da matéria, plasmas são macroscopicamente neutros e contêm um grande número de partículas ionizadas, apresentando comportamento coletivo devido às forças coulombianas de longo alcance [2].

Porém, nem todo meio contendo partículas carregadas pode ser chamado de plasma. Para que um conjunto de partículas interagentes possa ser classificado como tal, existem certas condições a serem satisfei$\operatorname{tas}[2-4]$ :

- Na ausência de perturbações externas, o plasma é macroscopicamente neutro. Em seu interior

\footnotetext{
${ }^{1}$ E-mail: costajr.e@gmail.com.

os campos microscópicos se cancelam, não existindo carga espacial líquida em uma região macroscópica.

- Condições de não-neutralidade macroscópica podem ocorrer apenas até distâncias da ordem do comprimento de Debye. Esse parâmetro físico é uma medida da distância ao longo da qual a influência do campo elétrico de uma partícula individual é sentida pelas outras partículas do plasma. As partículas carregadas se redistribuem de forma a blindar qualquer campo eletrostático dentro de uma distância da ordem do comprimento de Debye. Esse comprimento é dado por

$$
\lambda_{D}=\left(\frac{\epsilon_{0} k T}{n_{e} e^{2}}\right)^{\frac{1}{2}},
$$


onde $\epsilon_{0}$ é a permissividade elétrica no vácuo, $k$ é a constante de Boltzmann, $T$ é a temperatura, $n_{e}$ é a densidade eletrônica e $e$ é a carga fundamental. Para a existência de um plasma, obviamente, as dimensões físicas do sistema devem ser muito maiores que o comprimento de Debye.

- Por fim, outra importante característica dos plasmas é a estabilidade da neutralidade macroscópica de carga. Se um plasma é perturbado, os campos internos tendem a restabelecer a neutralidade inicial através de movimentos coletivos. Esses movimentos são caracterizados por uma frequência natural de oscilação, chamada frequência de plasma

$$
\nu_{p e}=\left(\frac{n_{e} e^{2}}{4 \pi^{2} m_{e} \epsilon_{0}}\right)^{\frac{1}{2}}
$$

onde $m_{e}$ é a massa do elétron. Essas oscilações coletivas são de alta frequência e, por isso, os íons são incapazes de acompanhar o movimento dos elétrons. Assim, os elétrons oscilam coletivamente ao redor dos íons pesados, sendo a força de restabelecimento exercida pelas interações coulombianas entre íons e elétrons. Colisões entre os elétrons e as partículas neutras, $\nu_{e n}$, tendem a amortecer essas oscilações e, para a definição de um plasma, a frequência de colisões deve ser menor que a frequência de plasma $\left(\nu_{c o l}<\nu_{p e}\right)$.

Devido à grande variedade de condições de plasmas contidos no universo, um grande número de tipos diferentes de ondas podem ser geradas. Como as distâncias entre suas fontes e a Terra são enormes, o estudo de ondas no meio interplanetário é de extrema importância para uma melhor compreensão dos processos físicos que ocorrem nas regiões geradoras. No fim das contas, as ondas transportam energia de uma região para outra, podendo ser usadas para se estudar as propriedades físicas das zonas em que foram geradas ou das regiões por onde passaram antes de serem detectadas.

Como os plasmas são eletricamente carregados e podem estar sujeitos a campos externos, o número possível de ondas mecânicas é maior que em um fluido normal, como por exemplo o ar, onde as ondas sonoras constituem o único tipo de onda possível [5].

Muito do que hoje se sabe sobre o Sol, o meio interplanetário e inúmeros outros corpos, tanto do nosso sistema solar quanto de outras galáxias, provêm de estudos de ondas emitidas por eles, uma vez que se torna impossível o envio de sondas e espaçonaves para a realização de medidas in situ.

Ondas que são geradas em meios onde a descrição MHD (magnetohidrodinâmica) é válida são chamadas ondas MHD, classificação que engloba as chamadas ondas de Alfvén.

\section{Equações e aproximações MHD}

O comportamento de fluidos ordinários difere consideravelmente do comportamento de sistemas de partículas carregadas. No entanto, certos conceitos e equações que foram desenvolvidos para descrever fluidos são aplicáveis a sistemas de partículas carregadas. Essa é a idéia básica da teoria MHD, que trata o plasma todo como um único fluido, sem considerar as espécies constituintes individualmente $[1,4,6]$.

\subsection{Teorias usadas para a descrição de plasmas}

Existem basicamente quatro abordagens teóricas diferentes para o estudo de plasmas: teoria orbital, teoria cinética, teoria de vários fluidos e teoria de um único fluido. Cada uma dessas teorias utiliza aproximações diferentes e se aplica a condições distintas de plasma.

A teoria orbital consiste em "seguir" o movimento individual de cada partícula na presença de campos elétricos e magnéticos aplicados externamente. Não são consideradas as variações causadas nos campos eletromagnéticos pelo movimento das próprias partículas, mas é útil para o estudo de plasmas pouco densos. Apesar de não se tratar de uma teoria de plasma propriamente dita, a teoria orbital é importante para a predição de efeitos físicos que conduzem a uma melhor compreensão dos processos dinâmicos que ocorrem em plasmas [2].

Para o estudo de plasmas muito densos, se faz necessária uma descrição estatística para a obtenção de uma representação macroscópica do mesmo. Na teoria cinética é necessário o conhecimento apenas da função de distribuição do conjunto de partículas a ser considerado, que contém todas as informações físicas relevantes sobre o sistema. A partir da resolução das equações cinéticas que regem a evolução da função de distribuição no espaço de fase, todas as quantidades físicas macroscópicas podem ser calculadas [2, 3, 5].

Se as colisões entre as partículas do plasma são muito frequentes, de modo que cada espécie possa manter uma função de distribuição local de equilíbrio, cada espécie pode ser tratada como um fluido diferente. Assim, o plasma pode ser tratado como uma mistura de dois ou mais fluidos imiscíveis, sendo a teoria chamada teoria de dois fluidos ou teoria de vários fluidos. Além das equações da eletrodinâmica, são usadas equações hidrodinâmicas que expressam as conservações de energia, massa e momentum para cada espécie constituinte do plasma $[1,2,4]$.

Por fim, existe a possibilidade de tratar o plasma todo como um único fluido, somando-se as equações sobre todas as espécies, através da teoria de único fluido. Uma forma simplificada dessa teoria, chamada aproximação MHD, é útil para o estudo de fenômenos de frequências muito baixas que ocorrem em plasmas altamente condutores imersos em campos magnéticos externos $[3,4]$. 


\subsection{Equações MHD}

$\mathrm{Na}$ teoria MHD as equações macroscópicas de transporte que são usadas na teoria de vários fluidos são generalizadas para o plasma como um todo. Assim, o plasma passa a ser descrito por essas equações, em adição às equações eletrodinâmicas, como um único fluido condutor, no limite de baixas frequências. As equações são equivalentes ao conjunto de equações para as espécies individuais de partículas, porém generalizadas coletivamente [1]. O tratamento MHD é muito útil em situações estacionárias ou de variação lenta, onde uma abordagem específica para cada uma das espécies seria muito complexa. Porém, se por um lado a teoria facilita o tratamento do plasma como um único fluido, por outro ocorre uma perda de informações sobre os seus constituintes específicos [4].

Entretanto, na prática as equações MHD dificilmente são empregadas em sua forma geral. Com base em algumas aproximações fisicamente justificadas, alguns termos das equações podem ser desconsiderados, gerando equações mais simples e mais fáceis de serem tratadas matematicamente. Uma das aproximações que é usada com frequência é a desconsideração do termo relativo à corrente de deslocamento $\left(\epsilon_{0} \partial \mathbf{E} / \partial t\right)$ na equação de Ampère [5]. Essa aproximação não é válida somente se o tempo característico de variação do campo elétrico for extremamente pequeno. Também costumase considerar que a neutralidade macroscópica é mantida com boa precisão, sendo a densidade elétrica igual a zero $(\rho=0)$. Por fim, aproxima-se a lei de Ohm pela sua forma generalizada, considerando-se desprezíveis suas derivadas temporais. Assim o conjunto de equações MHD simplificadas é constituído pelas seguintes equações [2]:

- equação da continuidade de massa

$$
\frac{\partial \rho_{m}}{\partial t}+\nabla \cdot\left(\rho_{m} \mathbf{u}\right)=0,
$$

onde $\rho_{m}$ é a densidade de massa e $\mathbf{u}$ é a velocidade média do plasma;

- equação de movimento

$$
\rho_{m} \frac{D \mathbf{u}}{D t}=\mathbf{J} \times \mathbf{B}-\nabla p,
$$

sendo $\frac{D}{D t}$ o operador derivada temporal total $\left(\frac{D}{D t}=\frac{\partial}{\partial t}+\mathbf{u} \cdot \nabla\right)$ e $\mathbf{J}$ a densidade de corrente elétrica ou fluxo de carga;

- equação da conservação da energia

$$
\nabla p=V_{s}^{2} \nabla \rho_{m},
$$

onde $V_{s}$ é a velocidade adiabática do som;
- equação de Ampére simplificada

$$
\nabla \times \mathbf{B}=\mu_{\mathbf{0}} \mathbf{J},
$$

e a equação de Faraday

$$
\nabla \times \mathbf{E}=-\frac{\partial \mathbf{B}}{\partial \mathbf{t}},
$$

ambas da eletrodinâmica, sendo $\mu_{0}$ a permeabilidade magnética do vácuo

- e por fim a lei de Ohm generalizada

$$
\mathbf{J}=\sigma_{0}(\mathbf{E}+\mathbf{u} \times \mathbf{B})-\frac{\sigma_{0}}{n e} \mathbf{J} \times \mathbf{B},
$$

onde $\sigma_{0}$ é a condutividade elétrica do plasma.

A condutividade térmica e a viscosidade são desconsideradas nesse conjunto de equações, sendo a diádica de pressão reduzida a uma pressão escalar na equação de conservação da energia. Além disso, a equação da continuidade da carga elétrica na ausência de mudanças na densidade macroscópica total está implícita na Eq. (6), já que $\nabla \times \mathbf{B}=\mu_{0} \mathbf{J}$ implica em $\nabla \cdot \mathbf{J}=\mathbf{0}$.

Costuma-se utilizar a aproximação ideal de um fluido perfeitamente condutor em situações onde a condutividade do plasma é muito alta. Nesse caso a Eq. (8) se reduz a

$$
\mathbf{E}=-\mathbf{u} \times \mathbf{B}
$$

com a condutividade elétrica do plasma $\left(\sigma_{0}\right)$ tendendo a infinito. O conjunto de equações MHD com a lei de Ohm expressa nessa forma é comumente chamado de equações MHD ideais.

\section{Ondas de Alfvén}

Ondas geradas em meios onde o tratamento MHD é válido são chamadas ondas MHD. Essas ondas são caracterizadas por baixas frequências, menores que a frequência de giro dos íons, e foram descobertas no início da era espacial [4]. Suas emissões são aumentadas durante períodos de vento solar perturbado, onde vários tipos de instabilidades podem se desenvolver no plasma.

O tipo de onda mais básico que se propaga em um fluido compressível e não-condutor são as ondas sonoras, que se propagam à velocidade do som. Porém, em um fluido compressível e condutor, imerso em um campo magnético externo, outros tipos de ondas são possíveis.

As ondas de Alfvén são ondas transversais que se propagam paralelamente ao campo magnético externo ( $\mathbf{k} \| \mathbf{B}$, onde $\mathbf{k}$ é o vetor de onda).

Sabe-se que a pressão magnética ao longo das linhas de campo é dada por $\mathbf{B}_{\mathbf{0}}^{\mathbf{2}} / \mu_{\mathbf{0}}$, sendo que as linhas se comportam como cordas elásticas submetidas a essa tensão. Em um fluido perfeitamente condutor, as partículas se comportam como se estivessem fixas às linhas de campo magnético. Portanto, de uma 
forma análoga às oscilações transversais em uma corda, espera-se que se um fluido condutor for perturbado de sua condição de equilíbrio, as linhas de campo oscilarão transversalmente. Essas ondas se propagam com a velocidade de Alfvén dada pela razão entre a tensão magnética e a densidade do meio [2]

$$
V_{A}=\left(\frac{\mathbf{B}_{\mathbf{0}}{ }^{2}}{\mu_{0} \rho_{m}}\right)^{\frac{1}{2}}
$$

A Fig. 1 mostra de forma esquemática a configuração das linhas de campo magnético na presença de ondas de Alfvén.

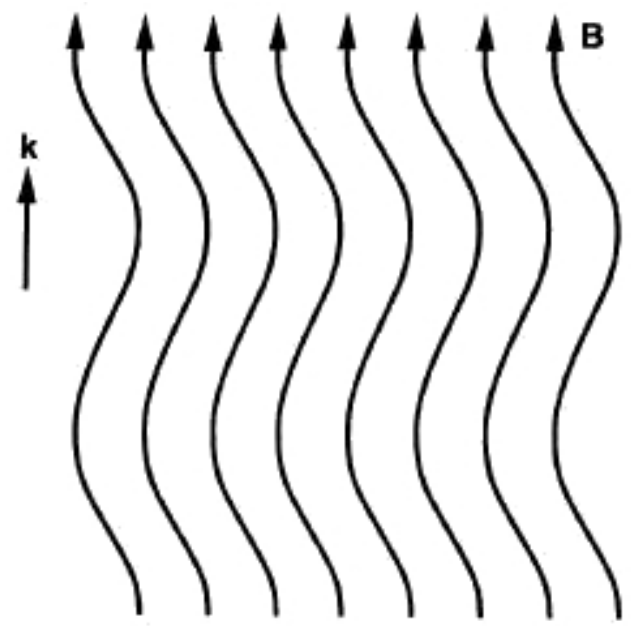

Figura 1 - Ondas de Alfvén transversais em um fluido condutor, compressível e magnetizado. A onda se propaga ao longo das linhas de campo, enquanto o movimento do fluido e as oscilações das linhas de campo se dão na direção perpendicular ao campo magnético [2].

Além das ondas de Alfvén, que são transversais, também podem ser geradas ondas longitudinais em fluidos MHD. Se o vetor de onda $\mathbf{k}$ e o movimento das partículas são paralelos ao campo magnético externo, as oscilações serão ondas sonoras comuns, uma vez que o movimento das partículas não será afetado pelo campo magnético. Se o vetor de onda $\mathbf{k}$ e o movimento das partículas forem perpendiculares a $\mathbf{B}_{\mathbf{0}}$, as partículas estarão sujeitas à pressão do fluido em adição à pressão magnética. Nesse caso, as ondas longitudinais são chamadas ondas magnetosônicas ou magnetoacústicas.

\section{Formalismo matemático das ondas de Alfvén}

Para o tratamento matemático das ondas MHD, são usadas basicamente as equações MHD apresentadas na Seção 2.2 .

Combinando as Eqs. (4), (5) e (6), chega-se a [2]

$$
\begin{array}{r}
\rho_{m} \frac{\partial \mathbf{u}}{\partial t}+\rho_{m}(\mathbf{u} . \nabla) \mathbf{u}=-V_{S}^{2} \nabla \rho_{m}+ \\
\frac{1}{\mu_{0}}(\nabla \times \mathbf{B}) \times \mathbf{B},
\end{array}
$$

e com o uso das Eqs. (7) e (9), obtem-se

$$
\nabla \times(\mathbf{u} \times \mathbf{B})=\frac{\partial \mathbf{B}}{\partial t} .
$$

Utilizando-se as seguintes equações para as perturbações no campo magnético e na densidade e velocidade do fluido, é possível deduzir as equações de dispersão (equação que relaciona $\omega$ e k) das ondas MHD

$$
\begin{gathered}
\mathbf{B}(\mathbf{r}, t)=\mathbf{B}_{0}+\mathbf{B}_{1}(\mathbf{r}, t), \\
\rho_{m}(\mathbf{r}, t)=\rho_{m 0}+\rho_{m 1}(\mathbf{r}, t), \\
\mathbf{u}(\mathbf{r}, t)=\mathbf{u}_{\mathbf{1}}(\mathbf{r}, t) .
\end{gathered}
$$

Substituindo-se as Eqs. (13), (14) e (15) nas Eqs. (3), (11) e (12), e desconsiderando termos de segunda ordem, obtém-se

$$
\begin{gathered}
\frac{\partial \rho_{m 1}}{\partial t}+\rho_{m 0}\left(\nabla \cdot \mathbf{u}_{1}\right)=0 \\
\rho_{m 0} \frac{\partial \mathbf{u}_{1}}{\partial t}+V_{S}^{2} \nabla \rho_{m 1}+\frac{1}{\mu_{0}} \mathbf{B}_{0} \times\left(\nabla \times \mathbf{B}_{1}\right)=\mathbf{0} \\
\frac{\partial \mathbf{B}_{1}}{\partial t}-\nabla \times\left(\mathbf{u}_{1} \times \mathbf{B}_{0}\right)=\mathbf{0}
\end{gathered}
$$

onde $\rho_{m 0}$ é a densidade do fluido em equilíbrio, a velocidade de equilíbrio é considerada nula $\left(\mathbf{u}_{0}=\mathbf{0}\right)$ e $\mathbf{B}_{0}$ é o campo ao longo do fluido, considerado uniforme e constante.

Combinando as Eqs. (16), (17) e (18), é possível obter uma expressão dependente apenas de $\mathbf{u}_{1}$. Para isso, diferenciando a Eq. (17) em relação ao tempo

$$
\begin{array}{r}
\rho_{m 0} \frac{\partial^{2} \mathbf{u}_{1}}{\partial t^{2}}+V_{S}^{2} \nabla\left(\frac{\partial \rho_{m 1}}{\partial t}\right)+ \\
\frac{1}{\mu_{0}} \mathbf{B}_{0} \times\left(\nabla \times \frac{\partial \mathbf{B}_{1}}{\partial t}\right)=0
\end{array}
$$

e usando as Eqs. (16) e (18), a Eq. (19) se torna

$$
\begin{array}{r}
\frac{\partial^{2} \mathbf{u}_{1}}{\partial t^{2}}-V_{s}^{2} \nabla\left(\nabla . \mathbf{u}_{1}\right)+ \\
\mathbf{V}_{A} \times\left\{\nabla \times\left[\nabla \times\left(\mathbf{u}_{1} \times \mathbf{V}_{A}\right)\right]\right\}=0
\end{array}
$$

onde $\mathbf{V}_{A}$ é o vetor velocidade de Alfvén, dado pela expressão $\mathbf{V}_{A}=\mathbf{B}_{0} / \sqrt{\left(\mu_{0} \rho_{m 0}\right)}$.

Considerando soluções de onda plana para a Eq. $(20), \mathbf{u}_{1}(\mathbf{r}, t)=\mathbf{u}_{1} \exp (i \mathbf{k} \cdot \mathbf{r}-i \omega t)$, os operadores $\nabla$ e $\frac{\partial}{\partial t}$ podem ser substituídos por $i \mathbf{k}$ e $-i \omega$, respectivamente, e a equação se torna

$$
\begin{array}{r}
-\omega^{2} \mathbf{u}_{1}+V_{S}^{2}\left(\mathbf{k} . \mathbf{u}_{1}\right) \mathbf{k}- \\
\mathbf{V}_{A} \times\left\{\mathbf{k} \times\left[\mathbf{k} \times\left(\mathbf{u}_{1} \times \mathbf{V}_{A}\right)\right]\right\}=\mathbf{0} .
\end{array}
$$


Por fim, usando a identidade vetorial $\mathbf{A} \times(\mathbf{B} \times \mathbf{C})=$ (A.C)B - (A.B)C, válida para quaisquer vetores, e rearranjando os termos, é possível obter

$$
\begin{array}{r}
-\omega^{2} \mathbf{u}_{1}+\left(V_{S}^{2}+V_{A}^{2}\right)\left(\mathbf{k} \cdot \mathbf{u}_{1}\right) \mathbf{k}+\left(\mathbf{k} \cdot \mathbf{V}_{A}\right)\left[\left(\mathbf{K} \cdot \mathbf{V}_{A}\right) \mathbf{u}_{1}-\right. \\
\left.\left(\mathbf{V}_{A} \cdot \mathbf{u}_{1}\right) \mathbf{k}-\left(\mathbf{k} \cdot \mathbf{u}_{1}\right) \mathbf{V}_{A}\right]=0 . \quad(22)
\end{array}
$$

A partir da Eq. (22), podem ser obtidas as soluções para ondas em magnetofluidos.

Para propagações paralelas ao campo magnético, o termo $\mathbf{k} . \mathbf{V}_{A}$ se reduz a $k V_{A}$, já que $\mathbf{k}$ e $\mathbf{V}_{\mathbf{A}}$ são paralelos nesse caso, e a Eq. (22) se torna

$$
\left(k^{2} V_{A}^{2}-\omega^{2}\right) \mathbf{u}_{1}+\left(\frac{V_{S}^{2}}{V_{A}^{2}}-1\right) k^{2}\left(\mathbf{u}_{1} \cdot \mathbf{V}_{A}\right) \mathbf{V}_{A}=\mathbf{0}
$$

Existem dois possíveis movimentos ondulatórios nesse caso, o modo longitudinal com $\mathbf{u}_{1}$ paralelo a $\mathbf{B}_{0}$ e $\mathbf{k}$ e o modo transversal, com $\mathbf{u}_{1}$ perpendicular a $\mathbf{B}_{0}$ e k.

Para o modo longitudinal, a partir da Eq. (23), temos

$$
\begin{aligned}
& \left(K^{2} V_{A}^{2}-\omega^{2}\right) \mathbf{u}_{1}+\left(\frac{V_{S}^{2}}{V_{A}^{2}}-1\right) k^{2} V_{A}^{2} \mathbf{u}_{1}=\mathbf{0}, \\
& \frac{\omega}{k}=V_{S}
\end{aligned}
$$

que corresponde a ondas sonoras. O campo magnético não influencia o movimento do fluido nesse caso.

Para o modo transversal, o termo $\mathbf{u}_{1} \cdot \mathbf{V}_{A}$ se torna nulo na Eq. (23), reduzindo a equação a

$$
\frac{\omega}{k}=V_{A},
$$

que é a velocidade de propagação das ondas conhecidas como ondas de Alfvén, em homenagem ao seu descobridor Hannes Alfvén.

É possível usar a Eq. (18) para a obtenção do campo magnético associado às ondas de Alfvén. Supondo soluções em ondas planas, a equação se torna

$$
-\omega \mathbf{B}_{1}-\mathbf{k} \times\left(\mathbf{u}_{1} \times \mathbf{B}_{0}\right)=\mathbf{0},
$$

e como $\left(\mathbf{u}_{1} \times \mathbf{B}_{0}\right) \perp \mathbf{u}_{1}$ e $\mathbf{k} \perp \mathbf{u}_{1}$, o termo $\mathbf{k} \times\left(\mathbf{u}_{1} \times \mathbf{B}_{0}\right)$ é na direção de $\mathbf{u}_{1}$, sendo o campo dado por

$$
\mathbf{B}_{1}=-\frac{B_{0}}{\omega / k} \mathbf{u}_{1}
$$

A Eq. (28) mostra que a perturbação no campo magnético é perpendicular ao campo magnético externo $\mathbf{B}_{0}$. A componente $\mathbf{B}_{1}$ adicionada à componente $\mathbf{B}_{0}$ dá às linhas de campo uma forma senoidal. A Fig. 2 mostra de forma esquemática a configuração do campo magnético devido à presença das perturbações causadas pelas ondas de Alfvén. Apesar do fluido e das linhas de campo magnético oscilarem, as ondas de Alfvén não provocam variações na densidade e na pressão do meio [2].

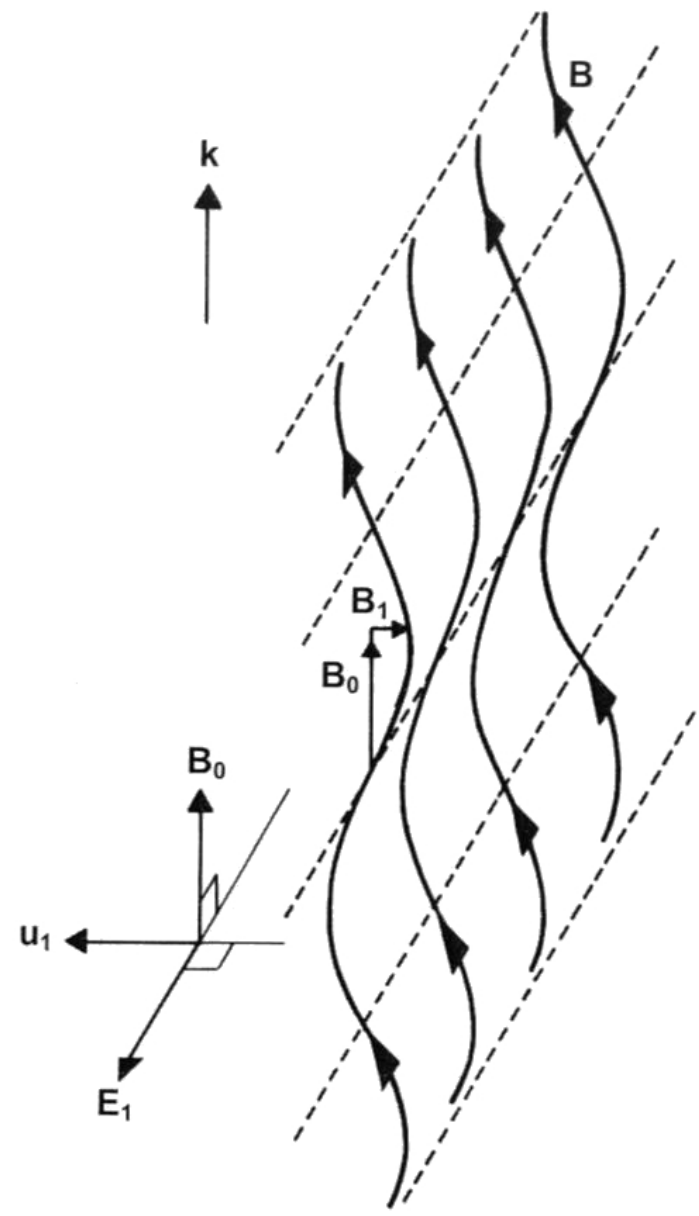

Figura 2 - Ilustração esquemática de ondas de Alfvén se propagando ao longo do campo magnético ambiente, mostrando as relações entre os parâmetros oscilantes [2].

\section{Ondas de Alfvén no meio interpla- netário}

A existência das ondas de Alfvén foi predita teoricamente por Hannes Alfven em 1942 [7]. Pouco tempo depois essas ondas foram detectadas em laboratório, primeiro em experimentos com fluidos magnetizados e posteriormente com plasmas magnetizados. Hoje em dia é conhecido o fato de que essas ondas são muito recorrentes, principalmente em plasmas espaciais, onde as condições MHD ideais são mais facilmente obtidas. Além disso, sua importância também se deve ao fato de que elas transportam energia entre diferentes regiões do espaço $[1,3]$. No vento solar elas são detectadas predominantemente se propagando em direções se afastando do Sol.

\subsection{Detecção das Ondas de Alfvén}

A partir do momento em que as medidas dos parâmetros de plasma e do campo magnético no meio interplanetário começaram a ser obtidas, ficou evidente que o vento solar é permeado por flutuações de baixas 
frequências e grandes amplitudes. Pela relação entre o campo magnético e a velocidade de fluxo do plasma, foi mostrado que essas flutuações são causadas por ondas de Alfvén [6,9].

A Fig. 3 mostra um exemplo dessas flutuações. Os três pares de curvas superiores mostram as componentes do campo magnético do vento solar $B_{N}, B_{T}$ e $B_{R}$ e as componentes da velocidade do vento solar $u_{N}, u_{T}$ e $u_{R}{ }^{2}$ O par de curvas inferior corresponde às intensidades totais do campo e da densidade. É possível notar que as componentes da velocidade e do campo magnético variaram, enquanto a magnitude total do campo e a densidade de plasma permaneceram aproximadamente constantes. Além disso, as variações nas componentes da velocidade e do campo magnético do vento solar foram bem correlacionadas entre si. Esse tipo de perturbação é característico das ondas de Alfvén [8].

A velocidade de propagação das ondas de Alfvén é bastante variável, a depender da região do espaço interplanetário onde esteja se propagando. Essa afirmação é até certo ponto óbvia, pois como pode ser constatado pela Eq. (10), a velocidade de propagação depende do campo magnético e da densidade de partículas do meio. Por exemplo, a velocidade de fase dessas ondas próximo ao Sol é extremamente alta $\left(\approx 1200 \mathrm{~km} \mathrm{~s}^{-1}\right.$ a cerca de 4 raios solares) se comparada à velocidade na órbita da Terra que é de aproximadamente $50-70 \mathrm{~km} \mathrm{~s}^{-1}$ [12].

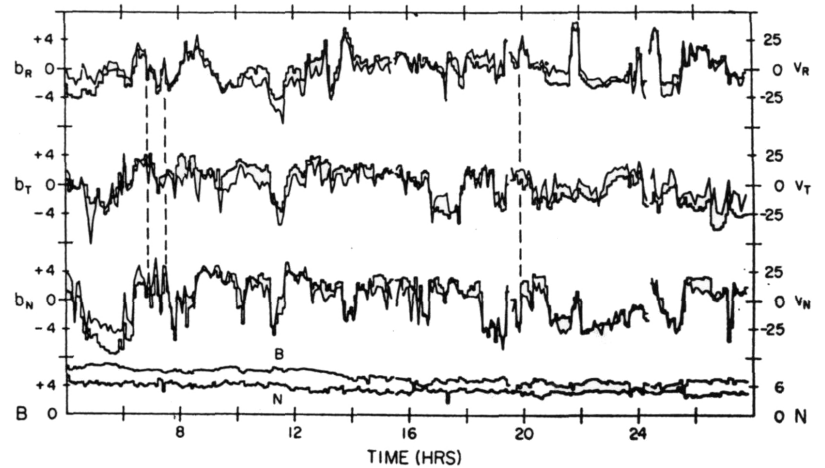

Figura 3 - Exemplo de ondas de Alfvén observadas no meio interplanetário. A densidade de prótons $\mathrm{N}$ e a magnitude do campo magnético permanecem aproximadamente constantes, ao passo que a intensidade de suas componentes varia constantemente [8].

\subsection{Ondas de Alfvén e atividade magnética}

A principal teoria para a determinação da entrada ou não de partículas e de energia do vento solar no interior da magnetosfera terrestre é a reconexão magnética.
Esse processo é caracterizado basicamente pela aniquilação de campos magnéticos antiparalelos, possibilitando assim a livre movimentação das partículas carregadas do vento solar $[5,13]$.

Como o campo magnético na parte diurna da magnetosfera é predominantemente na direção norte, se a direção do campo magnético interplanetário for, em contrapartida, predominantemente na direção sul, a probabilidade de reconexão é aumentada consideravelmente. Esses períodos de campo interplanetário sul podem ser causados por vários motivos diferentes, inclusive pelas flutuações magnéticas impostas pelas ondas de Alfvén.

Em um trabalho desenvolvido por Tsurutani e cols. [14], foi estudada a geoefetividade ${ }^{3}$ de flutuações Alfvênicas para a atividade magnética. O critério de seleção para as flutuações relacionadas às ondas de Alfvén foi que a correlação entre $V_{x}$ e $B_{x}$ fosse maior que 0,60 . O principal resultado obtido foi que o campo magnético sul produzido por períodos alfvênicos é equivalentemente efetivo ao campo sul produzido por períodos não-alfvênicos. Assim, a análise estatística mostrou que, quanto à geoefetividade, não há diferenças se a estrutura causadora de campo sul for uma onda de Alfvén ou se for qualquer outro tipo de estrutura interplanetária [14].

Em outros dois estudos clássicos, Gonzalez e Tsurutani [15] e Tsurutani e Gonzalez [16] descobriram e classificaram os eventos que foram chamados de HILDCAAs (do inglês High-Intensity Long-Duration Continuous AE Activity). Esses eventos são caracterizados por acontecerem fora da fase principal de tempestades geomagnéticas, com picos no índice $\mathrm{AE}^{4}$ acima de 1000 nT, durando no mínimo dois dias e desde que o índice AE não atinja valores inferiores a $200 \mathrm{nT}$ por períodos superiores a 2 horas. Nesses trabalhos foi mostrado que as HILDCAAs estão intimamente relacionadas às flutuações magnéticas associadas às ondas de Alfvén no meio interplanetário, delegando assim a essas ondas o status de geoefetivas $[15,16]$.

A Fig. 4 mostra o resultado de correlação cruzada obtido por Gonzalez e Tsurutani [15] para as componentes sul das perturbações no campo magnético interplanetário e da velocidade do vento solar. A partir da alta correlação obtida para lag zero, essas estruturas causadoras das HILDCAAs foram prontamente identificadas como ondas de Alfvén. Ainda nesse trabalho, vários outros eventos de atividade geomagnética foram identificados como resultantes de flutuações alfvênicas, apesar de não serem classificados como HILDCAAs pelos restritivos critérios de classificação.

\footnotetext{
${ }^{2}$ Direções referentes ao sistema de coordenadas heliosféricas RTN. R aponta radialmente do Sol para a espaçonave, T aponta na direção $\Omega \times R$, onde $\Omega$ é o eixo de spin do Sol e N completa o sistema destrógiro.

${ }^{3} \mathrm{~A}$ geoefetividade de uma estrutura interplanetária é relacionada à capacidade da estrutura gerar atividade geomagnética no interior da magnetosfera terrestre [17,18], ou seja, de causar perturbações nos sitemas de corrente e campo magnético do planeta. Tais distúrbios podem provocar danos a sistemas tecnológicos no espaço e na superfície, como por exemplo, os sistemas de comunicação.

${ }^{4} \mathrm{O}$ índice $\mathrm{AE}$ mede em zonas aurorais a atividade geomagnética causada pela entrada de partículas do vento solar na magnetosfera terrestre.
} 


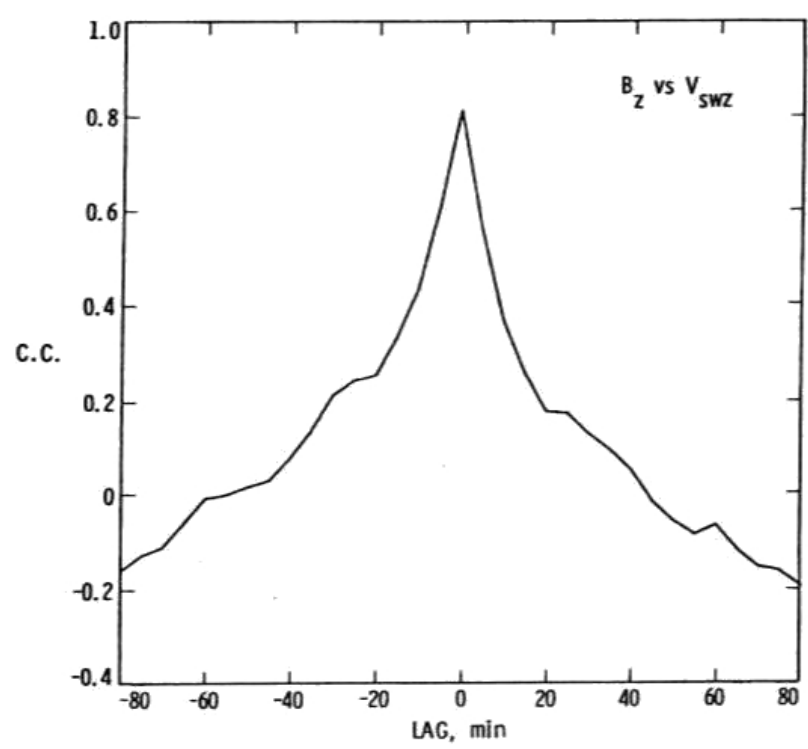

Figura 4 - Correlações cruzadas para os eventos classificados como HILDCAAs, mostrando a predominância das ondas de Alfvén.

Por fim, Tsurutani e cols. (1994) mostraram que a taxa de ocorrência de descontinuidades interplanetárias é muito alta quando ondas de Alfvén são detectadas. As descontinuidades interplanetárias são identificadas por mudanças nítidas na direção e/ou magnitude do campo magnético [19]. Isso corrobora ainda mais a idéia da alta geoefetividade das ondas de Alfvén.

\subsection{Possíveis processos de amortecimento das ondas de Alfvén}

Como era de se esperar, as ondas de Alfvén não se propagam indefinidamente, sofrendo amortecimento à medida que se propagam em regiões de diferentes regimes de plasma e de campo magnético. Os processos que levam a esse amortecimento ainda não são bem conhecidos, sendo ainda motivo de muita discussão. Porém, algumas indicações já foram descobertas sobre o assunto.

Uma das formas de amortecimento das ondas de Alfvén é o espalhamento de partículas, onde a onda é amortecida ao ceder parte de sua energia às partículas $[12,20]$. Porém, muito pouco se sabe sobre os detalhes dessas interações ondas-partículas. Estudos mais específicos sobre o assunto poderão esclarecer quantitativamente como esse processo ocorre.

Uma outra forma de amortecimento das ondas está relacionado ao fato de que essas ondas levam à aceleração perpendicular ao campo magnético de partículas, que através de efeitos diamagnéticos causariam os decréscimos magnéticos. ${ }^{5}$ Supostamente, esses decréscimos são causados pela força ponderomotiva ${ }^{6}$ associada à aceleração perpendicular das partículas.
Essa força está associada aos gradientes de campo da onda [12]. Porém, ainda não existem confirmações de que esse seja realmente o processo decisivo para o amortecimento das ondas. Simulações computacionais da relação entre as ondas de Alfvén e os decréscimos magnéticos podem ser decisivas para se definir se esse processo é realmente eficiente no que diz respeito ao amortecimento das ondas.

Em suma, muito pouco se sabe sobre os processos que levariam ao amortecimento das ondas de Alfvén. Aliás, muito pouco se sabe sobre as próprias ondas. Uma melhor compreensão dos processos de geração ainda é almejada. Os conhecimentos obtidos até hoje se baseiam muito ainda em observações. Futuramente, dados observacionais mais detalhados e simulações computacionais ainda mais específicas provavelmente ajudarão a preencher várias das lacunas existentes sobre a geração, propagação e amortecimento dessas ondas.

\section{Referências}

[1] L.F. Burlaga, Interplanetary Magnetohydrodynamics (Oxford University Press, 1995).

[2] J.A. Bittencourt, Fundamentals of Plasma Physics (Springer-Verlag, Berlin, 2004), $3^{\text {rd }}$ ed.

[3] D.A. Gurnett and A. Bhattacharjee, Plasma Physics and Controlled Fusion (Cambridge University Press, England, 1984).

[4] F.F. Chen, Introduction to Plasma Physics (Plenum Press, New York, 1984).

[5] M.G. Kivelson and C.T. Russell, Introduction to Space Physics (Cambridge University Press, Boston, 1995).

[6] G.K. Parks, Physics of Space Plasmas: An Introduction (Addison-Wesley Publishing Company, 1991).

[7] H. Alfvén, Nature, 150, 405 (1942).

[8] J.W. Belcher and L. Davis, Journal of Geophysical Research 76, 3534 (1971).

[9] J.W. Belcher, L. Davis and E.J. Smith, Journal of Geophysical Research 74, 2302 (1969).

[10] E.N. Parker, Planetary and Space Science 9,461 (1962).

[11] K.S. O Filho and M.F. Saraiva, Astronomia e Astrofísica (Editora da Universidade Federal do Rio Grande do Sul, Porto Alegre, 2000).

[12] B.T. Tsurutani, G.S. Lakhina, J.S. Pickett, F.L. Guarnieri, N. Lin and B.E. Goldstein, Nonlinear Processes in Geophysics 12, 321- (2005).

[13] S.I. Akasofu and S. Chapman, Solar Terrestrial Physics (Oxford University Press, Inglaterra, 1997).

[14] B.T. Tsurutani, T. Gould, B.E. Goldstein, W.D. Gonzalez and M. Sugiura, Journal of Geophysical Research 95, 2241 (1990).

[15] W.D. Gonzalez and B.T. Tsurutani, Planetary and Space Science 35, 1101 (1987).

\footnotetext{
${ }^{5}$ Decréscimos Magnéticos (MDs, do inglês Magnetic Decreases) são regiões do espaço e das magnetosferas planetárias onde o campo magnético apresenta decréscimos consideráveis, podendo ser reduzido a até 10\% de seu valor ambiente [20,21].

${ }^{6} \mathrm{~A}$ força ponderomotiva está associada aos gradientes de campo da onda, que levam a uma variação da energia cinética das partículas ao longo de uma órbita ciclotrônica [4].
} 
[16] B.T. Tsurutani and W.D. Gonzalez, Planetary and Space Science 35, 405 (1987).

[17] M.V. Alves, E. Echer and W.D. Gonzalez, Journal of Geophysical Research 111, A07S05 (2006).

[18] E. Echer, W.D. Gonzalez and M.V. Alves, Space Weather 4, S06001 (2006).

[19] B.T. Tsurutani, C.M. Ho, E.J. Smith, M. Neugebauer, B.E. Goldstein, J.S. Mok, J.K. Arballo, A. Balogh, D.J.
Southwood and W.C. Feldman, Geophysical Research Letters 21, 2267 (1994).

[20] B.T. Tsurutani, G.S. Lakhina, O.P. Verkhoglyadova, E. Echer and F.L. Guarnieri, Nonlinear Process in Geophysics 17, 1 (2010).

[21] E. Costa Jr., E. Echer, M.V. Alves, B.T. Tsurutani, F.J.R. Simões Jr., F.R. Cardoso and G.S. Lakhina, Journal of Atmospheric ans Solar-Terrestrial Physics, doi:10.1016/jastp.2011.01.022 (2011). 